EOMmUn Communication et organisation

Ory

Structure et communication

\title{
Les trois mac
}

Chaké Matossian

\section{OpenEdition}

Journals

Édition électronique

URL : http://journals.openedition.org/communicationorganisation/1597

DOI : 10.4000/communicationorganisation. 1597

ISSN : 1775-3546

\section{Éditeur}

Presses universitaires de Bordeaux

\section{Édition imprimée}

Date de publication : 1 novembre 1992

ISSN : 1168-5549

\section{Référence électronique}

Chaké Matossian, "Les trois mac », Communication et organisation [En ligne], 2 | 1992, mis en ligne le 26 mars 2012, consulté le 19 avril 2019. URL : http://journals.openedition.org/

communicationorganisation/1597 ; DOI : 10.4000/communicationorganisation.1597

Ce document a été généré automatiquement le 19 avril 2019

(C) Presses universitaires de Bordeaux 


\title{
Les trois mac
}

\author{
Chaké Matossian
}

1 L'homme machine a alimenté la fable et la philosophie, il pénètre aujourd'hui le quotidien, sous-tend de diverses façons l'image de l'homme contemporain, tantôt boule informe, tantôt projeté dans la machine ou retrouvant une source cathartique dans un héros sportif. De l'androgyne d'Aristophane à l'Unheimliche hoffmanno-freudien, en passant par La Mettrie, ce rapport ambivalent, cet entrelacement de la répétition mécanique et du vivant a essentiellement été envisagé sur le mode métaphorique. Il semble cependant que le rapport du corps avec la machine repose tout autant sur un processus métonymique, que nous tenterons d'éclairer par trois exemples, trois types d'hommes-machines contemporains, qui ressortissent à trois types de mac, Macdonald's, Mc Enroe et Macintosh.

D’emblée, le préfixe patronyme, "Mac» manisfeste une valeur auto-référentielle et métonymique, en ce que, d'une part, il signifie homme, « man », et que, d'autre part, une fois le contexte connu (alimentation, sport, informatique), dans les trois cas dont il est ici question, le préfixe suffit à signifier l'entièreté du nom. Tout le monde comprend à demimot, ce qui montre le pouvoir communicationnel et universalisant que détient ce demi nom, «mac », l'homme. Ce pouvoir communicationnel se trouve en outre intensifié par le fait que le terme "mac » ne manque pas d'évoquer, tant en français qu'en anglais, un certain genre de commerce - qui se rapporte justement aux corps - et, plus généralement, la vente ${ }^{1}$.

\section{Macdonald's : la fabrique des corps}

3 Les Macdonald's forment une chaîne internationale qui se soutient d'un travail à la chaîne et se développe sur différents plans : spatial, organique, rituel, esthétique. Macdonald's s'installe partout, mais pas n'importe où. Il marque son territoire, tatoue l'espace, avec son logotype, un grand $\mathrm{M}$ jaune aux formes arrondies se détachant sur un fond rouge. Extérieurement, le Macdonald's fonctionne comme point de repère et de rencontre, il devient le familière l'étranger, le connu dans l'inconnu. Même si on le déteste, on sait 
qu'il ne recèle pas de surprise, il propose, partout, une nourriture informe, de façon rapide et pour un prix modique (ou se voulant tel). Le décor intérieur adopte en général le mélange ou la juxtaposition des deux couleurs primaires du logotype, qui trouve encore son équivalent chromatique dans la juxtaposition du pain (jaune) et de la viande (rouge) et dans ce qui accompagne la plupart du temps cet aliment de base, la frite (jaune) et le ketchup (rouge). L'ensemble de toutes ces composantes primaires, comme les couleurs jaune et rouge, ou le pain et la pomme de terre, ou même la sonorité du mot «mac", colorée de rouge $^{2}$, crée un environnement sensoriel primaire ou primitif, en correspondance avec un corps (re)devenu primaire. Un corps à la fois éveillé et apaisé, d'une part, par la luminosité des couleurs et, d'autre part, par la substance spongieuse du hamburger qui correspond, en termes cartésiens, à la modération, c'est-à-dire aussi, à la moyenne, soit encore, à ce qui ressortit è la masse. La modération caractérise chaque sens, elle existe dans les couleurs, la musique et l'alimentation: « entre les couleurs, la verte qui consiste en l'action la plus modérée (qu'on peut nommer par analogie la proportion d'un à deux), est comme l'octave entre les consonances de la musique, ou le pain entre les viandes que l'on mange, c'est-à-dire celle qui est la plus universellement agréable $»^{3}$. Macdonald's offre donc le plaisir de la modération dans l'aliment spongieux mais compense l'inertie découlant de l'apaisement excessif par le choix des couleurs vives, stimulantes, en accord tant avec le besoin de vitesse qu'avec le plaisir infantile. Le corps dans un Macdonald's ne perçoit que deux couleurs chaudes qui lui signalent le seul but de sa présence, la combustion des aliments, leur cuisson dans l'estomac. Les formes arrondies du logotype, le caractère fonctionnel des chaises moulantes et des tables glissantes, collées ensemble et collant de façon inamovible au corps, forment un tout agglutiné, une masse, la version agrandie du hamburger. Et le corps en ingurgitant les aliments de base, farineux et féculent, se transmute peu à peu en hamburger, apparait comme un morceau de viande enveloppé d'un gros pain mou. Un corps informe, un noncorps.

4 La présence du corps se trouverait transférée dans le hamburger, version industrielle et immanentisée de l'hostie que chacun attend, en file, de recevoir des mains de l'officiant. C'est sans doute en tablant sur la rapidité que Macdonald's parvient a l'efficacité et à l'effectivité du monde religieux, le rythme du service calquant ici la performativité de l'énonciation de la formule eucharistique ${ }^{4}$. Seule une extrême vitesse autorise Macdonald's à rivaliser avec le monde de la transcendance, seule la performance abolit le temps de la transformation et permet une mise en présence instantanée. Ceci est votre corps. Les clients de Macdonald's communient, ils s'auto et s'entredévorent, pour régénérer sans fin la grande masse informe. Au demeurant, c'est le même terme, fast, qui indique la vitesse et la performance du système («fast food») et qui, dans la sphère religieuse, signifie l'abstinence. Le caractère sacré du Macdonald's se trouve en outre renforcé par le nom même qui se rapporte à une maladie et au pouvoir guérisseur du toucher magique attribué aux families Macdonald's ${ }^{5}$.

5 Dans cet univers primaire et primitif subtilement ritualisé et sacralisé, le Macdonald's joue en quelque sorte le rôle du «mageiros» grec. Le «mag des Anciens assurait simultanément les fonctions de "boucher-cuisinier sacrificateur", dont l'arme, la «makhaira ", l'égorgeoir ${ }^{6}$ faisait l'objet d'un rituel visant à le faire disparaitre, à l'occulter. Le mac d'aujourd'hui rassemble les masses, il présente, de façon rituelle et universelle, une viande dont l'aspect informe et la texture spongieuse vient à faire oublier au consommateur qu'il intègre son corps. Avec le hamburger, plat, rond, dégoulinant, il 
ne faut plus de couteau pour manger la viande, plus de couverts. Le décor aseptisé et moderne, l'efficacité industrielle, cachent donc, sous le masqué de la civilisation, l'abolition des manières de table et le retour d'une violence orgiaque ou sacrificielle présente dans le contact direct et primitif qui s'établit désormais entre le corps et la nourriture.

\section{Mc Enroe : la machine dévorée}

Mc Enroe se démarque des autres joueurs de tennis en ce qu'il est caractérisé par l'excès. Si d'autres vedettes du monde du tennis peuvent accéder à la singularité par leur performance ou leurs extravagances, Mc Enroe reste le seul être décrit comme un enfant terrible, un « wild kid ». Il déborde, un peu comme le hamburger et va, comme ce dernier, offrir à l'homme contemporain l'image et l'atmosphère du moi infantile. D'emblée, et ce n'est sans doute pas par hasard, le tennisman s'est vu attribuer le nom du célèbre hamburger, «Big Mac ». Grâce à ses excès, Mc Enroe dépasse le statut de l'homme et du sportif, il laisse éclater en lui quelque chose qui déborde, une force qui est celle de la colère et qui rappelle l'attitude d'un enfant, aux antipodes du « fairplay ». Mc Enroe, c'est l'homme au stade primaire, le héros (la sonorité du nom «Enroe » nous convie à faire cette association), un héros colérique et infantile qui exhibe son échec, trahit sa vanité blessée et se révèle, par la-même, comme un " casseur de raquettes "; ainsi qu'on le disait au XVII ${ }^{e}$ siècle ${ }^{7}$. Ce tempérament colérique engendre une certaine sympathie, car, de tous temps, ou du moins depuis Aristote, le colérique fait montre de bonté, de vivacité et son âme se détache de toute servilité8. Pour Descartes également, l'extériorisation rapide de la colère signale la bonté de l'individu, sa capacité d'amour'. Et si Kant ne paraît pas apprécier beaucoup le tempérament colérique, ce type d'homme «ardent » et instable, dominé par le «désir des honneurs ${ }^{10}$, il le rattache néanmoins à l'esthétique du sublime: "Toute affection du genre vigoureux (celui qui éveille la conscience de nos forces, de vaincre toute résistance) (animi strenui) est esthétiquement sublime, par exemple la colère, ou même le désespoir (le désespoir révolté, non pas le désespoir découragé) $\aleph^{11}$. Mc Enroe, héros sportif, est davantage qu'une machine musclée, une «fabrica » du XXe siècle. Il réunit les qualités de son exces tempéramental et détient, en tant que "casseur de raquettes ", un pouvoir cathartique essentiel permettant au public de satisfaire ses pulsions agressives infantiles ${ }^{12}$. Et si le wild kid agresse son public, celuici le lui rend bien en transformant le match en source cathartique, en tragédie, au cours de laquelle elle dévore son héros Big Mac.

\section{Macintosh : la divine machine}

7 Le macintosh ne se veut pas un ordinateur comme les autres. S'il est vrai que chaque marqué prétend, à travers la publicité, détenir des qualités propres, il reste que Macintosh est le seul à faire appel à un critère de distinction, à s'imposer comme machine supérieure. Le «Mac » est incompatible au contraire de toutes les autres marques et c'est cette incompatibilité qui le rend absolument autre, qui lui permet d'échapper à la banalité, à la médiocrité. Incompatible, il en devient incomparable. Le logotype d'Apple, une pomme polychrome et déjà mordue, joue sur de multiples significations. Plus qu'une marque, il signale un monde et assimile subtilement l'utilisateur à une divinité portant ou transportant le monde miniaturisé ${ }^{13}$. La pomme évoque aussi le fruit de l'arbre défendu et 
nous entraîne à associer le Macintosh à la séduction, de même qu'à la connaissance et à une sorte d'orgueil, source d'une force prométhéenne qui nous porte a défier les dieux. L'entame, la trace de morsure, révèle que la machine, loin d'être inaccessible, intouchable, a été au contraire manipulée, mordue, et que celui qui en aura goûté acquerra une force nouvelle, meilleure. La morsure opère un retour, celui qui aura croqué du mac en sera mordu. La pomme, équivalent du fruit de l'arbre de la science, en appelle a l'image du paradis - connotation consolidée par le spectre des couleurs- elle vise à montrer que le mac n'est pas l'outil prolongeant les facultés de l'homme mais qu'il est incorporé par l'utilisateur. Et que celui-ci ne peut en sortir indemne : " La femme vit que l'arbre était bon à manger et séduisant a voir, et qu'il était, cet arbre, désirable pour acquérir le discemement. Elle prit de son fruit et mangea. Elle en donna aussi à son man, qui était avec elle, et il mangea. Alors leurs yeux è tous deux s'ouvrirent et ils connurent qu'ils étaient nus $»^{14}$

Un macintosh n'est pas un ordinateur, il est un macintosh, un mac, c'est à dire une chose personnifiée, un élément vivant, hors du commun, avec lequel l'utilisateur fusionne et sur lequel il se projette, ainsi qu'en témoignent les annonces publicitaires de Mac-Apple. Avec son mac, l'homme voit enfin qu'il était nu. Il devient un mec.

\section{NOTES}

1. The Conciae Oxford Dictionary, «Mac » see Mack. « Mack »:« pirrep, procurer » (abréviation de mackerel qui vient du néerlandais « makelaer », courtier).

2. Jakobson, Roman Langage enfantin et aphasie. Paris, Flammarion, 1980, p. 88 : «On constate également une très nette propension à relier les voyelles les plus chromatiques aux couleurs les plus franches, en particulier au rouge... ».

3. Descartes, L'homme. Euvres philosophiques, Paris, Garnier, 1963, vol. I, pp. 424-425.

4. Sur la question du temps de l'énonciation dans la formule eucharistique, nous renvoyons à Louis Marin, La parole mangée, Paris, Méridiens Klinck-sieck 1986, p.31:« ...pour cette proposition "ceci est mon corps» et pour elle seulement, le temps de l'énociation (avec son passé (eed) et son futur (mon corps) avant et «pre » le " est», présent central de la présence) génère le présent réel que décrit renonce «ceci est mon corps»: le temps de l'énondation, entame infime et infinie de la présence, produit la présence réelle du Corps divin dans l'indiqué de « ceci », qu'il détruit ».

5. The English Dialect Dictionary, Ed. by Joseph Wright, Oxford University Press, 1961. "Macdonald's disease »: An affection of the lungs. It is called the Macdonald's disease because there are particular tribes of Macdonald'a who are helieve to cure it with the charme of their touch and the use of certain set of word There must be no fee given of any kind.

6. Detienne, Marcel, «Pratique » culinaire » et esprit de sacrifice ", in La cuisine du sacrifice en pays grec. Paris, Gallimard, 1979, p. 21.

7. Furetière Antoine; Dictionnaire universel, La Haye et Rotterdam, chez Amout et Reinier Leen, 1690. Article «Maquereau : ce mot, aussi bien que celui de Maquignon, vient de maque, qui en vieux français signifie vente ». 
8. Aristote, Éthique de Nico-maque V., chap. V : « les Irascibles s'emportent promptement et plus qu'il ne faut contre des personnes et pour des causes qui n'en valent pas la peine. Cependant eet emportement tombe rapidement et c'est ce qu'il y a peut-être en eux de meilleur ».

9. Descartes, Les Passions de l'âme, art. 201

10. Kant, Anthropologie du point de vue pragamatique. Parat, Vrin. 1970 pp. 137-138.

11. Kant, Critique de la faculté de juger. Pari », Vrin. 1968, p. 109.

12. Freud, "Au-delà du principe de plaisir », in Essais de psychanalyse, Paris,

Payot, p. 18 « Noussavons d'ailleurs que les enfant » expriment souvent des impulsions hostiles analogues en rejetant des objets qui à leurs yeux, symbolisent certaines personnes ».

13. La pomme sert d'image représentant l'univers réduit et portatif, ainsi que l'a montré George Poulet dans Las Métamorphoses du cercle. Paris, Garnier Flammarion, 1979, p. 74.

14. Genèse, 3-9,7.

\section{RÉSUMÉS}

S'interrogeant sur le rapport contemporain du corps à la machine, l'auteur trouve dans trois types de «Mac» célèbres trois façons pour le corps de se transformer en machine, non seulement sur le mode métaphorique et par Identification (le patronyme "Mac» signifiant homme et fils de) mais surtout sur le mode métonymique par des phénomènes d'entredévoration (immanentisation de la transcendance, récupération de l'orgiaque et de la tragédie) et de contiguité (l'homme devenant le prolongement de la machine).

\section{AUTEUR}

\section{CHAKÉ MATOSSIAN}

Docteur en philosophie et théorie de la communication. Titre de la thèse : « Les représentations de la maladie-analyse d'une théorie de la communication à partir de l'œuvre de La Mettrie ». Thèse annexe : «Étude sur la représentation du corps (le cas du règlement des funiculaires de Lisbonne) ». Argumentateur : Louis Marin. Actuellement professeur à l'Université Nouvelle de Lisbonne ; enseigne la philosophie de la communication et l'épistémologie au département de Communication. Coordinatrice adjointe du département de communication, responsable des programmes Erasmus, présidente du centre d'Études de communication et languages. Ecrit dans des revues portugaises et internationales. 